\title{
Long-term bond strength and endogenous enzymatic activity of a chlorhexidine-containing commercially available adhesive
}

\author{
Tatjana Maravića ${ }^{\text {, Allegra Comba }}{ }^{\mathrm{a}}$, Sandra Ribeiro Cunha ${ }^{\mathrm{b}}$, Valeria Angeloni ${ }^{\mathrm{c}}$, Milena Cadenaro ${ }^{\mathrm{d}}$, \\ Erika Visinitini ${ }^{\mathrm{e}}$, Chiara Ottavia Navarra ${ }^{\mathrm{f}}$, Stefano Salgarello ${ }^{\mathrm{g}}$, Lorenzo Breschi ${ }^{\mathrm{a}, *}$, \\ Annalisa Mazzoni ${ }^{\text {a }}$ \\ ${ }^{a}$ Department of Biomedical and Neuromotor Sciences, DIBINEM, University of Bologna - Alma Mater Studiorum, Via San Vitale 59, 40125, Bologna, Italy \\ ${ }^{\mathrm{b}}$ Department of Restorative Dentistry, School of Dentistry, University of São Paulo, Av. Prof. Lineu Prestes 2227, 05508-000, São Paulo, Brazil \\ ${ }^{\mathrm{c}}$ Private Practice, Via Martiri della Foce 20, 17031, Albenga, Italy \\ ${ }^{\mathrm{d}}$ Department of Medical Sciences, University of Trieste, Piazza dell'Ospitale 1, 34129, Trieste, Italy \\ ${ }^{\text {e }}$ S.C. Clinica di Chirurgia Maxillofacciale e Odontostomatologia AsuiTS, Piazza dell'Ospitale 1, 34129, Trieste, Italy \\ ${ }_{\mathrm{f}}^{\mathrm{f}}$ Department of Medical, Surgical and Health Sciences, University of Trieste, Piazza dell'Ospitale 1, 34129, Trieste, Italy \\ ${ }^{\mathrm{g}}$ Dipartimento di Specialità Medico-Chirurgiche, Scienze Radiologiche e Sanità Pubblica - Università degli Studi di Brescia, P.le Spedali Civili 1, 25123, Brescia, Italy
}

\section{A R T I C L E I N F O}

\section{Keywords:}

Chlorhexidine

Dental bonding systems

Hybrid Layer

Aging

Dentin

\begin{abstract}
A B S T R A C T
Objectives: The aim of this study was to investigate, by the means of microtensile bond strength ( $\mu \mathrm{TBS}$ ) test, gelatin and in situ zymography, the influence of $0.2 \%$ CHX contained within a commercially available adhesive on long-term bond strength and endogenous enzymatic activity.

Methods: Non-carious teeth were subjected to $\mu$ TBS test ( $N=15$ for each group) and stressed until failure. $\mu$ TBS was evaluated immediately and after 12-month storage in artificial saliva at $37^{\circ} \mathrm{C}$. Dentin powder was obtained from additional teeth $(\mathrm{N}=7)$ for gelatin zymography, while for in situ zymography, 3 teeth for each group were selected. Gelatin and in situ zymography were performed in dentin powder and slices of dentin, respectively, to assess the ability of $0.2 \% \mathrm{CHX}$ blended within the adhesive to inhibit endogenous enzymatic activity.

Results: $\mu$ TBS bond strength was higher in the CHX-containing groups, immediately as well as after aging. The bond strength significantly decreased after 12-month aging. The activation of endogenous MMPs was found to be related to the presence of $\mathrm{CHX}$ within the adhesive system and the bonding strategy employed.

Conclusions: Under this perspective $0.2 \%$ CHX blended within Peak Universal adhesive monomer seems to increase immediate bond strength, to preserve bond strength over time and to efficiently inhibit endogenous enzymatic activity in dentin. Hence, blending the CHX in low concentrations within the adhesive could be recommended as a feasible technique in every-day clinical practice.

Clinical significance: Using CHX-containing adhesives could be recommended due to several benefits: it seems to increase the longevity of the hybrid layer; the inhibitor appears to be efficiently delivered to the dentinal substrate and to inhibit endogenous enzymatic activity, without prolonging chair time.
\end{abstract}

\section{Introduction}

In spite of rapid development in the field of dental materials, the issue of time affected degradation of the hybrid layer has still not been resolved. Dentinal endogenous enzymes, such as MMPs and cysteine cathepsins can accelerate the aging process of the hybrid layer by degrading the collagen fibrils exposed after certain dental procedures [1-3]. MMPs are $\mathrm{Zn}^{2+}$ - and $\mathrm{Ca}^{2+}$-dependent enzymes with the capability of degrading almost all components of the dentinal extracellular matrix [4]. So far, several MMPs have been identified in dentin (MMP-2, -3, -8, -9 and -20) [5-9]. These enzymes are active during the development of dentin [10], while later in life, they stay trapped within the mineralized dentin and are inactive. However, they can be reactivated by adhesive procedures or caries [11]. Furthermore, the synergistic effect of the dentinal MMPs and cysteine cathepsins is thought to contribute to the this process [12].

\footnotetext{
* Corresponding author.

E-mail addresses: tatjana.maravic2@unibo.it (T. Maravić), allegra.comba@unibo.it (A. Comba), sandra.cunha@gmail.com (S.R. Cunha), angeloni.valeria@gmail.com (V. Angeloni), mcadenaro@units.it (M. Cadenaro), E.VISINTINI@fmc.units.it (E. Visinitini), c.navarra@fmc.units.it (C.O. Navarra), stefano.salgarello@unibs.it (S. Salgarello), lorenzo.breschi@unibo.it (L. Breschi), annalisa.mazzoni@unibo.it (A. Mazzoni).
} 
Several approaches have been investigated to improve the preservation of the hybrid layer. Among these is the use of MMPs inhibitors, such as chlorhexidine (CHX), galardin, tetracycline, bisphosphonates, quaternary ammonium compounds [13]. CHX, a widely used antimicrobial agent in dentistry, is one of the most investigated MMP inhibitors, due to its ability to inhibit MMP-2, -8 and 9 in very low concentrations (total inhibition of MMP-2 at a CHX concentration as low as $0.0001 \%$, MMP-9 at $0.002 \%$ and MMP- 8 at $0.02 \%$ CHX) [14], as well as cysteine cathepsins [15]. It is assumed that the ability of CHX to inhibit MMPs derives from its binding properties [4,14]. CHX has been investigated in numerous in vitro and in vivo studies, used as either as a separate primer in water solution [16,17], or incorporated in one of the components of etch-and-rinse (E\&R) or self-etch (SE) adhesive systems, within the primer [18-20] or the adhesive [21-25] agent.

So far, CHX showed mixed outcomes. Several authors found beneficial effect of the use of CHX strength of the adhesive layer [16-18,21-23,26-28], while others found no difference between the treated and untreated groups [24,25,29-31]. These studies differ in methodology, materials used, as well as aging time, which could be the cause of the discrepancies among the available results. CHX water solution used as a separate primer has shown good results. However, there has been a tendency to avoid the use of CHX as a separate primer since it prolongs chair-time, but rather to incorporate it into one of the components of the adhesive system. Apart from the experimental systems used in in vitro studies, an adhesive system containing $0.2 \% \mathrm{CHX}$ has been released on the market for clinical use (Peak Universal Bond, Ultradent Products Inc., South Jordan, UT).

Hence, the aim of this study was to investigate, by the means of microtensile bond strength test ( $\mu \mathrm{TBS}$ ), gelatin and in situ zymography, the influence of $0.2 \% \mathrm{CHX}$ contained within Peak Universal Bond (PUB), applied in E\&R or self-etch SE mode, on the preservation of aged hybrid layers.

The null hypotheses tested were: (1) no differences in immediate bond strength exist between the tested groups, (2) presence of CHX within the adhesive system does not affect the stability of the tested adhesive interfaces after 12 -month-ageing in artificial saliva at $37^{\circ} \mathrm{C}$, (3) activation of endogenous MMPs is not related to the presence of CHX within the adhesive system or the bonding strategy.

\section{Materials and methods}

\subsection{Microtensile ( $\mu \mathrm{TBS}$ ) bond strength test}

Non-carious molars ( $\mathrm{N}=15$ for each group) stored in $0.5 \%$ chloramine in water at $4{ }^{\circ} \mathrm{C}$ were used within 1 month after extraction. The occlusal enamel was removed using a low speed diamond saw under water irrigation (Micromet, Remet, Bologna, Italy) and dentin was exposed to create a flat surface for conventional bonding. Specimens were assigned to treatment groups and bonded according to manufacturer's instructions (Table 1):

1 Dentin + Peak Universal Bond (PUB, Ultradent, South Jordan, UT, USA) adhesive system in E\&R mode (containing 0.2\% CHX)

2 Dentin + Adper Scotchbond Universal (SBU, 3 M ESPE, St. Paul, $\mathrm{MN}, \mathrm{USA}$ ) adhesive system in the E\&R mode - control

3 Dentin + PUB adhesive system in SE mode (containing $0.2 \%$ CHX)

4 Dentin + SBU adhesive system in SE mode - control

Further, 4-mm thick resin composite build-ups were created (Filtek Z250, 3 M ESPE) and polymerized for $40 \mathrm{~s}$ using a light-emitting diode light-curing unit. Non-trimming technique [32] was used to obtain resin-dentin sticks with cross-sectional area of approximately $0.9 \mathrm{~mm}^{2}$. Each stick was measured, and the dimensions recorded for bond strength calculation. Within each of the groups, the sticks were equally divided into two parts and stored at $37^{\circ} \mathrm{C}$ in artificial saliva prepared in accordance with Pashley et al. [1], for $24 \mathrm{~h}\left(\mathrm{~T}_{0}\right)$ or for 12 months $\left(\mathrm{T}_{12}\right)$.
After aging, $\mu$ TBS sticks were stressed until failure using a simplified universal testing machine at a crosshead speed of $1 \mathrm{~mm} / \mathrm{min}$ (Bisco Inc., Schaumburg, IL, USA). The evaluation of failure modes and classification as cohesive (C), adhesive (A), or mixed (M) failures was done according to Breschi et al. [16]. Although recorded, the number of prematurely debonded sticks per each group was not included in the statistical analysis, due to the fact that all premature failures occurred during the cutting procedure, which was performed at time zero and did not exceed $3 \%$ of the total number of tested specimens.

\subsection{Gelatin zymography}

The zymographic essay on dentin extracts was performed according to the protocol of Mazzoni et al. [33] to investigate the expression of MMP-2 and -9. Enamel, cement and pulp were removed from 7 sound human molars and dentin was reduced to powder using a Retsch mill (Model MM400, Retsch GmbH, Haan, Germany). The powder from all teeth was mixed together and a pool of dentin powder was created. Aliquots of dentin powder were divided into 6 groups: G1 - mineralized dentin powder (control); G2 - demineralized dentin powder treated with $10 \mathrm{wt} \%$ phosphoric acid for $10 \mathrm{~min}$ (control); G3 - demineralized as G2 and treated with Peak Universal Bond for $30 \mathrm{~min}$; G4 - demineralized as G2 and treated with SBU for 30 min; G5 - mineralized dentin powder treated with Peak Primer for 30 min followed by Peak Universal Bond application for $30 \mathrm{~min}$; G6 - mineralized dentin powder treated with SBU for $30 \mathrm{~min}$. The groups treated with the adhesives or primer were subjected to a series of three suspensions in $1 \mathrm{~mL}$ of acetone followed by centrifuges (20.800xg for $20 \mathrm{~min}$ ), to properly remove the adhesive resin [34]. Further, the specimens were resuspended in the extraction buffer $(50 \mathrm{mM}$ Tris- $\mathrm{HCl} \mathrm{pH} \mathrm{6,} \mathrm{containing}$ $5 \mathrm{mM} \mathrm{CaCl} 2,100 \mathrm{mM} \mathrm{NaCl}, 0.1 \%$ Triton $\mathrm{X}-100,0.1 \%$ nonionic detergent P-40, $0.1 \mathrm{mM} \mathrm{ZnCl} 2,0.02 \% \mathrm{NaN} 3$ ) and kept for $24 \mathrm{~h}$ at $4{ }^{\circ} \mathrm{C}$ under constant agitation, after which they were sonicated for $10 \mathrm{~min}$ (at $\approx 30$ pulses $)$ and centrifuged $\left(20.800 \mathrm{~g} ; 20 \mathrm{~min} ; 4^{\circ} \mathrm{C}\right)$. The supernatant was separated and re-centrifuged two times in order to remove traces of the powder. The protein content was concentrated by centrifugation in Vivaspin concentrators (10,000 kDa cut-off; Vivaspin Sartorius Stedim Biotech, Goettingen, Germany) for $30 \mathrm{~min}$ at $25^{\circ} \mathrm{C}(15,000 \mathrm{~g}, 3$ times $)$ and the protein concentration was determined using Bradford assay (Bio-Rad, Hercules, CA, USA). The extracted protein aliquots were diluted in Laemmli sample buffer in a 4:1 ratio. Sodium dodecyl sulfatepolyacrylamide gel electrophoresis (SDS-PAGE, 10\%) was performed under non-reducing conditions. The gel contained $1 \mathrm{mg} / \mathrm{mL}$ of fluorescently labelled (2-methoxy-2,4-diphenyl-3(2 H)-furanone) gelatin. Pre-stained low-range molecular weight SDS-PAGE standards (Bio-Rad) were used as molecular-weight markers. After electrophoresis, the gels were washed for $1 \mathrm{~h}$ in $2 \%$ Triton X-100, incubated in activation solution $(50 \mathrm{mmol} / \mathrm{L}$ Tris-HCl, $5 \mathrm{mmol} / \mathrm{L} \mathrm{CaCl} 2, \mathrm{pH} 7.4)$ for $48 \mathrm{~h}$ and photographed under long wavelength ultraviolet light illumination (Gel Doc XR System, Bio-Rad). The zymographic assay was performed and analyzed in triplicate.

\subsection{In-situ zymography of resin-dentin interfaces}

One-millimeter-thick slabs of middle/deep dentin were obtained from extracted human third molars $(\mathrm{N}=3)$ using the low-speed Micromet saw with water-cooling. Each slab was further divided into four parts to test all the investigated groups on the same substrate (Fig. 1). Silicon-carbide paper (600-grit) was used to create a standardized smear layer on each dentin surface. One surface of each quarter of a slab was treated with the adhesive systems as described for $\mu$ TBS test. This was followed by a 1-mm build-up with flowable composite (Filtek 250 flow; $3 \mathrm{M}$ ESPE); the composite was polymerized for $40 \mathrm{~s}$ using a light-emitting diode light-curing unit (Curing Light 2500; 3 M ESPE). After completion of those procedures, the bonded assemblies were sectioned vertically into 1 -mm-thick specimens to expose the adhesive- 
Table 1

Components, compositions, and application procedure of the tested adhesives (information supplied by the manufacturer).

\begin{tabular}{|c|c|c|}
\hline Adhesive system & Composition & Manufacturer's instructions \\
\hline $\begin{array}{l}\text { Peak Universal Bond (Ultradent } \\
\text { Products Inc., South Jordan, UT) }\end{array}$ & $\begin{array}{l}\text { 1. Peak SE Primer: ethyl alcohol, methacrylic acid, 2-hydroxyethyl } \\
\text { methacrylate } \\
\text { 2. Peak Universal Bond resin: ethyl alcohol, 2-hydroxyethyl Methacrylate, } \\
\text { Methacrylic Acid, Chlorhexidine di(acetate) } 0.2 \%\end{array}$ & $\begin{array}{l}\text { ER: } \\
\text { 1. Apply etchant ( } 35 \% \text { phosphoric acid) for } 15 \mathrm{~s} \\
\text { 2. Rinse for } 5 \mathrm{~s} \\
\text { 3. Air dry } 2 \mathrm{~s} \\
\text { 4. Apply a puddle coat of the adhesive and gently agitate } \\
\text { for } 10 \mathrm{~s} \text { (Peak Universal Bond) or } 20 \mathrm{~s} \text { (SBU) } \\
\text { 5. Dry } 10 \mathrm{~s} \text { using } 1 / 4 \text { to } 1 / 2 \text { air pressure (Peak Universal } \\
\text { Bond) or } 5 \mathrm{~s} \text { (SBU) } \\
\text { 6. Light polymerize for } 10 \mathrm{~s} \text { at } 1200 \mathrm{~mW} / \mathrm{cm} 2\end{array}$ \\
\hline $\begin{array}{l}\text { a'Scotchbond Universal (3M ESPE, } \\
\text { Neuss, Germany) }\end{array}$ & $\begin{array}{l}\text { 2-hydroxyethyl methacrylate, bisphenol a diglycidyl ether dimethacrylate } \\
\text { (BISGMA), decamethylene dimethacrylate, ethanol, water, silane treated } \\
\text { silica, 2-propenoic acid, 2-methyl-, reaction }\end{array}$ & $\begin{array}{l}\text { SE: } \\
\text { 1. Initial use of Peak SE Primer requires activation of the } \\
\text { two components } \\
\text { separated in the syringe } \\
\text { 2. Application of the Peak SE Primer with microbrush for } \\
20 \mathrm{~s} \text { using continuous scrubbing on dentin. Do not scrub } \\
\text { enamel. } \\
\text { 3. Thin/dry for } 3 \mathrm{~s} \text { using air/water } \\
\text { syringe or high-volume suction directly over preparation } \\
\text { 4. Apply a puddle coat of the adhesive and gently agitate } \\
\text { for } 10 \mathrm{~s} \text { (Peak Universal Bond) or } 20 \mathrm{~s} \text { (SBU) } \\
\text { 5. Dry } 10 \mathrm{~s} \text { using } 1 / 4 \text { to } 1 / 2 \text { air pressure (Peak Universal } \\
\text { Bond) or } 5 \mathrm{~s} \text { (SBU) } \\
\text { 6. Light polymerize for } 10 \mathrm{~s} \text { at } 1200 \mathrm{~mW} / \mathrm{cm}^{2}\end{array}$ \\
\hline
\end{tabular}

${ }^{\mathrm{a}}$ In the SE mode, the procedure starts from step 4.

dentin interfaces. Each specimen was glued to a microscope slide, ground down approximately to the thickness of $50 \mu \mathrm{m}$ and polished.

In-situ zymography was performed following the protocol reported by Mazzoni et al. [35]. Briefly, self-quenched fluorescein-conjugated gelatin mixture (E-12055; Molecular Probes, Eugene, OR, USA) was placed on the specimen covering the polished resin-dentin surfaces and then protected with a coverslip. The specimens were incubated for $12 \mathrm{~h}$ at $37^{\circ} \mathrm{C}$ in a humid chamber avoiding direct contact with water, or exposure to light. Confocal laser scanning microscope was used to examine the specimens after incubation (excitation wavelength, $488 \mathrm{~nm}$; emission wavelength, $530 \mathrm{~nm}$; Model A1-R; Nikon, Tokyo, Japan). For each assembly, a series of images were made (one image per each $1 \mu \mathrm{m}$ into the depth of the sample) to show the hydrolysis of the quenched fluorescein-conjugated gelatin substrate, presented as green fluorescence. ImageJ software (National Institutes of Health, Bethesda, MD, USA) was used to measure integrated density of the fluorescence signals. The differences in the intensity of the fluorescence between the experimental and the control groups were used as a relative measurement of the differences in the enzymatic activity of the hybrid layer between the tested groups.

\subsection{Statistical analysis}

$\mu$ TBS test results were analyzed using the two-way ANOVA test followed, when significant, by pair-wise comparisons using the Tukey Test. Since the in situ zymography data failed to comply with normality requirements (Shapiro-Wilk test), Kruskal Wallis test was used to compare the density of the fluorescence signal within the different investigated groups. The significance threshold was set at $\mathrm{p}<0.05$. All the analyses were performed in Sigma plot v. 12.0 (Systat Software Inc.).

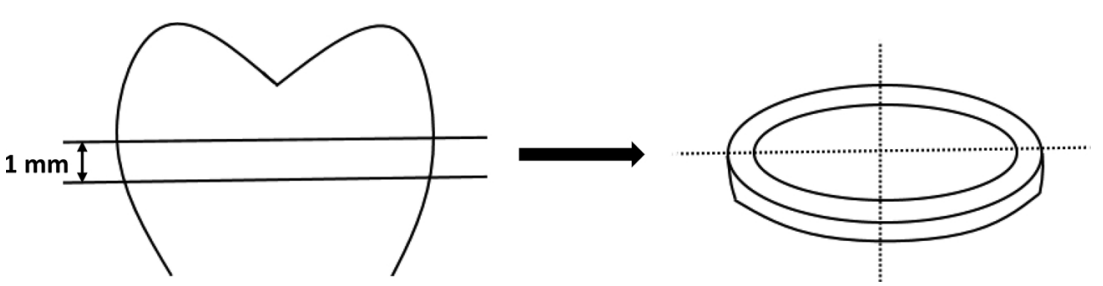

Fig. 1. Schematic overview of tooth cutting procedure for in situ zymography, making sure that all the groups are tested on the same substrate. 
Table 3

Percentages of failure modes after microtensile test.

\begin{tabular}{lll}
\hline Adhesive system & $\mathrm{T}_{0}$ & $\mathrm{~T}_{12}$ \\
\hline PUB E\&R (0.2\%CHX) & $35 \% \mathrm{~A}$ & $45 \% \mathrm{~A}$ \\
SBU E\&R (control) & $65 \% \mathrm{M}$ & $55 \% \mathrm{M}$ \\
& $50 \% \mathrm{~A}$ & $50 \% \mathrm{~A}$ \\
PUB SE (0.2\%CHX) & $50 \% \mathrm{M}$ & $50 \% \mathrm{M}$ \\
& $35 \% \mathrm{~A}$ & $60 \% \mathrm{~A}$ \\
SBU SE (control) & $5 \% \mathrm{CD}$ & $40 \% \mathrm{M}$ \\
& $65 \% \mathrm{M}$ & \\
& $30 \% \mathrm{~A}$ & $45 \% \mathrm{~A}$ \\
& $10 \% \mathrm{CD}$ & $55 \% \mathrm{M}$ \\
\hline
\end{tabular}

Fractures were classified as: A, adhesive; $\mathrm{CD}$, cohesive failure in dentin; CC, cohesive failure in resin composite; $\mathrm{M}$, mixed failure.

after 12 months, but not significantly.

Failure mode distribution of the de-bonded specimens is shown in Table 3. The predominant failure mode was the mixed, followed by the adhesive failure mode in all the tested groups.

\subsection{Gelatin zymography}

Gelatinolytic activity of the investigated groups is shown in Fig. 2a. Mineralized dentin powder (G1; Lane 1) showed week enzymatic activity in the corresponding to the molecular weights of MMP-2 and -9. Demineralized dentin extract (G2; Lane 2) showed multiple forms of gelatinolytic enzymatic activity, with the weak MMP-2 active and proform at $66 \mathrm{kDa}$ and $72 \mathrm{kDa}$, as well as a stronger signal of MMP-9 active and pro-forms at $86 \mathrm{kDa}$ and $92 \mathrm{kDa}$, respectively. The active enzyme forms were more pronounced. In both E\&R and SE Peak groups (Lanes 3 and 5, respectively), there is an absence of the activity of MMP-2, while the activity of MMP-9 seems to be reduced. On the other hand, enzymatic activity in the E\&R and SE SBU groups is more pronounced compared to the controls (Lane 4 and 6, respectively). Quantification of enzymatic activity is shown in Fig. $1 \mathrm{~b}$ and corresponds to the qualitative results.
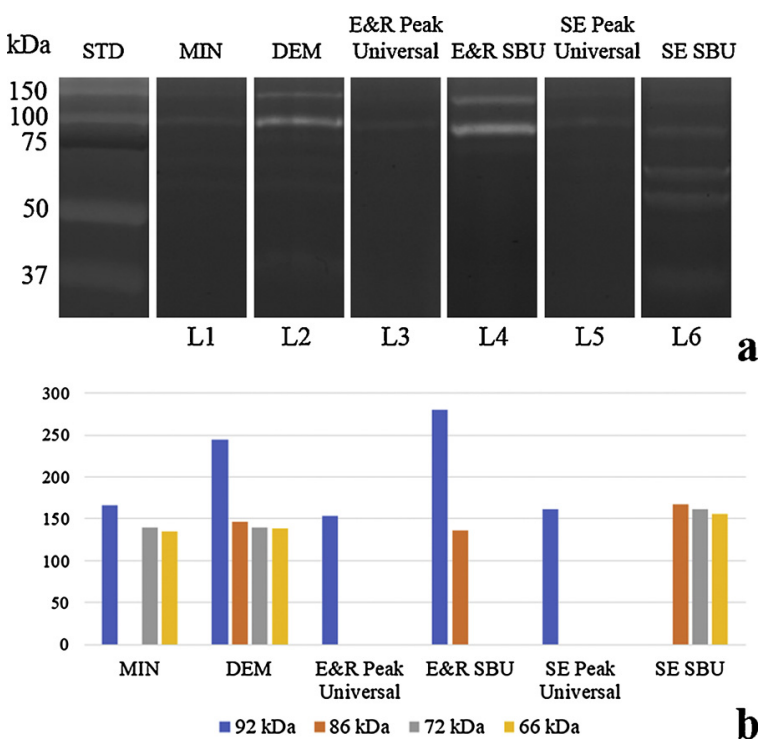

Fig. 2. (a) Gelatin zymography: Figure showing the differences in the enzymatic activity between the investigated groups presented as light bands in the area of the molecular weights of pro- and active MMP-2 and MMP-9; (b) Graph illustrating the densitometric evaluation of bands obtained from the zymographic analysis of proteins extracted from dentin powder. STD = Standard; $M I N=$ Mineralized dentin powder; $D E M=$ Demineralized dentin powder; $E \& R$ $=$ Etch-and-Rinse Mode; $S E=$ Self-Etch Mode; $S B U=$ Scotchbond Universal.

\subsection{In situ zymography}

The results obtained on the confocal microscope revealed differences in the fluorescence signal exhibited by different tested groups (Fig. 3). The level of enzymatic activity corresponds to the density of the green fluorescence signal (Fig. 3a-d). Quantification of the density of the fluorescence (Fig. 3i) corresponded to qualitative findings and demonstrated a reduction in enzymatic activity in the hybrid layers created with PUB used in the E\&R mode compared to the control SBU used in the same mode $(p<0.05)$. Both adhesives used in the SE mode showed a similar level of fluorescence $(p>0.05)$, while they exhibited lower enzymatic activity compared to the same adhesives used in $\mathrm{E} \& \mathrm{R}$ mode. Interestingly, Peak used in the E\&R mode showed a similar activity as SBU used in SE mode.

\section{Discussion}

The results of the presented study showed higher immediate bond strength in the experimental compared to control groups. Hence, the first null hypothesis should be rejected. The bond strength after 12month-aging was lower compared to the immediate bond strength, but significantly higher in the CHX-containing groups compared to the controls, which requires the rejection of the second hypothesis. The activation of endogenous MMPs was found to be related to the presence of CHX within the adhesive system and the bonding strategy, which supports the rejection of the third hypothesis.

Since the hybrid layer is composed of organic dentinal matrix, the remnants of hydroxyapatite, resin monomers, and solvents, the changes in any of these components can influence the longevity of resin-dentin bonds [36]. Neither E\&R nor SE adhesives are able to fully infiltrate the collagen network, creating different degrees of infiltration in different depths of the hybrid layer [13]. This leaves part of the collagen fibrils exposed and prone to degradation over time. The degradation of the hybrid layer can mainly be contributed to the hydrolysis of the resin and/or collagen fibrils and to the disorganization of the collagen fibrils [37]. Pashley et al. were the first to show that endogenous dentinal enzymes can degrade exposed collagen fibrils over time in the absence of bacteria, and that this effect can be diminished by the use of CHX [1]

CHX has an excellent substantivity to dentin [38] and it binds to mineralized as well as demineralized dentin [39]. It is well known that low concentrations of CHX can inhibit MMPs and cysteine cathepsins $[14,15]$. Several authors established the beneficial effect of CHX water solution used as a primer in the preservation of the $\mu$ TBS $[16,17,26,27,40]$. On the other hand, certain authors found no influence of CHX water solution on bonding performance [29], or a negative influence on mechanical properties of the hybrid layer after the use of this inhibitor [41]. Nevertheless, the majority of the available research is in favor of the use of CHX. In every-day dental practice, it is of great importance for patients, as well as dental practitioners, to make chairtime as short as possible. Therefore, there is a tendency to avoid additional steps in the restorative procedure, thus efforts have been made to incorporate $\mathrm{CHX}$ within one of the components of bonding systems. Since adhesives act like semipermeable membranes [16], it was assumed that the adhesive layer would serve as a reservoir of CHX, released into the hybrid layer over time, which could contribute to the durability of the adhesive bond.

Therefore, in the present study, the authors tested a commercially available adhesive containing $0.2 \%$ CHX. The resin system containing $0.2 \%$ CHX revealed significant improvement in bond stability, immediately, as well as after aging. After a 12-month storage in artificial saliva, a general decrease in bond strength was noted, however, the CHX-containing groups showed high bond strength values, in fact, higher than the $\mathrm{T}_{0}$ values in the control group. Failure modes found in this study are relatively evenly distributed between mixed and adhesive one, with the mixed mode being the most prevalent in all investigated groups, regardless of the aging. This means that the failures usually 
E\&R Peak Universal

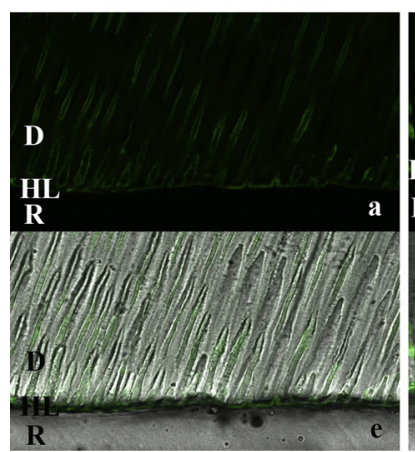

E\&R SBU

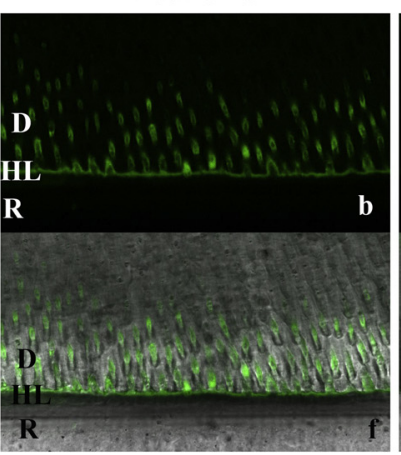

SE Peak Universal

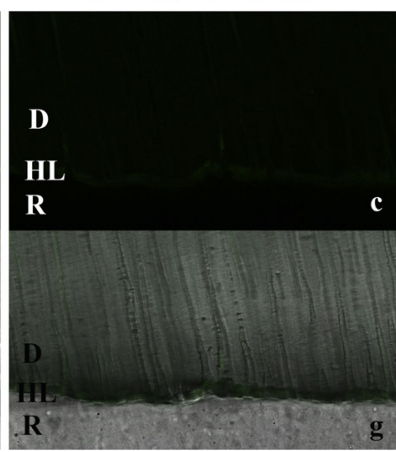

SE SBU

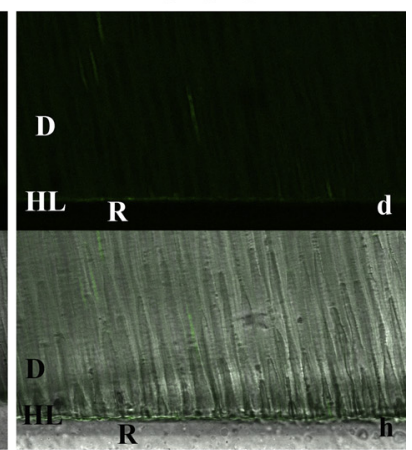

60

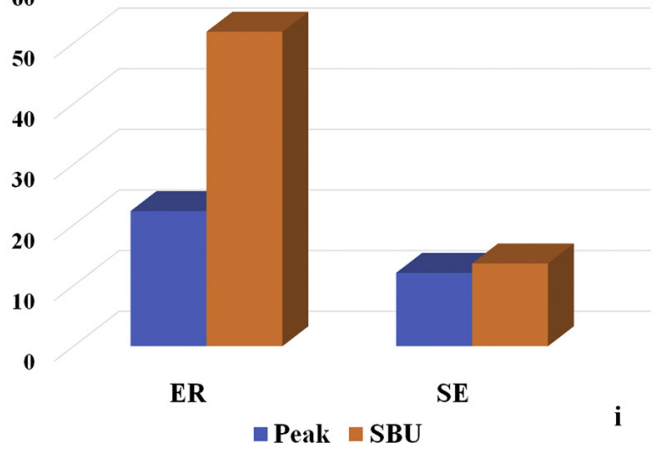

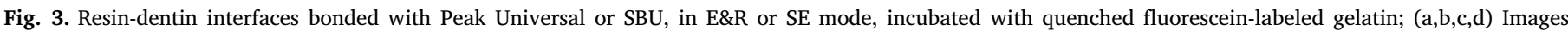

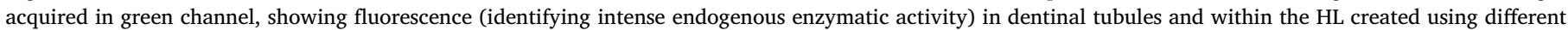

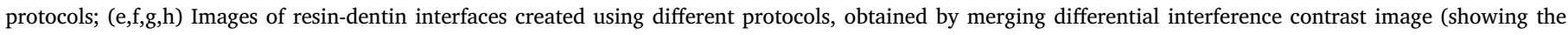

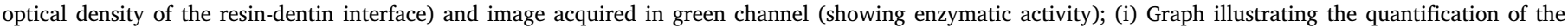

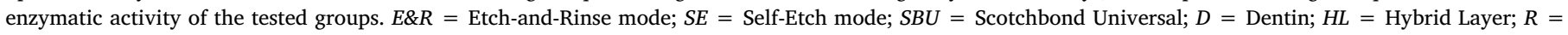
Resin Composite. (For interpretation of the references to colour in this figure legend, the reader is referred to the web version of this article).

started in the composite and propagated through the adhesive layer into dentin. These results differ from the results of a similar recent study [25] in which the same commercial adhesive system was used as the experimental group. Sabatini et al. found no difference between the control and experimental group in shear bond stress, regardless of aging (6 months), or the bonding mode (E\&R/SE). Further, Sabatini et al. [25] confirmed the efficacy of CHX in inhibiting the dentinal MMPs using a zymographic assay testing only the effect of $\mathrm{CHX}$ aqueous solution. This finding is in accordance with our results, however, in our study, gelatin zymography was for the first time performed following all the steps required by the manufacturers for the use of Peak Universal and SBU systems in E\&R and SE mode. Hence, the results of Sabatini et al. [25] are not fully comparable to our study, due to the different methodology, control group and aging time. Perhaps the aging of 6 months was not long enough to reveal differences between the tested groups. Furthermore, the control group in the study of Sabatini et al. was Peak LC Bond, which is a material similar to PUB, since they are produced by the same manufacturer, with the difference that Peak LC Bond does not contain $0.2 \%$ CHX. Since a different system was used as the control group in the present study (SBU), the possibility that PUB is superior to SBU as an adhesive system and that the differences are not driven by the presence of CHX cannot be excluded. However, since the present study did not only test the $\mu \mathrm{TBS}$, but also showed, using two different zymographic assays, that the PUB system presents lower dentinal enzymatic activity compared to SBU, especially in the E\&R mode, and having in mind previous studies that have shown preservation of the hybrid layer and collagen matrix with the use of CHX, we could hypothesize that there is a correlation between the CHX within PUB and better bond strength. Indeed, future studies should include observation of the bonded interfaces under a transmission electron microscope to investigate whether there are differences in the preservation of the collagen matrix between the tested groups and validate the results of the present study.

The results of other studies with CHX incorporated into one of the parts of the adhesive system varied, especially in SE mode. Certain studies showed that the incorporation of CHX within adhesive blends, used in the E\&R mode could contribute to the preservation of bond strength after a 12-month water storage [21,22]. Yiu et al. [23] found mixed results where the effect of CHX was correlated with the composition of the adhesive used in the E\&R mode. Zhou et al. found that the incorporation of $0.1 \%-1 \%$ CHX into a SE primer could preserve dentin bond strength after a 12-month aging [18]. On the other hand, a study incorporating $2 \% \mathrm{CHX}$ into a SE adhesive found no influence of CHX on bonding performance after 6 months [24]. It is thought that acid etching during E\&R procedures can denaturize enzymes within the etched dentin, due to the low $\mathrm{pH}$ values of the phosphoric acid. However, Mazzoni et al. [2] showed that enzymatic activity in dentin could be reactivated after acid etching. The authors hypothesized that acidetching inactivates the superficial layer of the enzymes, while activating the residual latent enzymes within the underlying demineralized dentin. The SE adhesives, on the other hand, have a $\mathrm{pH} 1.5-2.7$, which means that the more acidic adhesives could denaturize the enzymes, while the ones with a higher $\mathrm{pH}$ value could contribute to the activation of enzymatic activity [3]. Moreover, CHX binds to calcium chloride released from dentinal tissue by the influence of acids (especially the primers of SE adhesives) [13,14], which could diminish the inhibitory effect of CHX. Furthermore, in the SE systems, the hybrid layer is created simultaneously as the acidic monomer etches into the dentin. Therefore, it is assumed that there are less denuded collagen fibrils at the bottom of the hybrid layer compared to the E\&R system, and consequently less amount of activated MMPs [33]. Perhaps this is why CHX might have less influence on dentinal enzymatic activity in SE 
adhesives. This corresponds to the in situ zymography results of the present study, since the activity in the SE groups was much lower compared to the E\&R groups, regardless of the presence of $\mathrm{CHX}$ in the bonding system. All the aforementioned differences could be responsible for the discrepancies in the results found in different studies investigating E\&R and SE dentin bonding strategies.

Further, the aging time used in this study could be considered short, and in vitro and clinical studies with a longer monitoring time should be conducted to assess the efficacy of this adhesive system. However, the clinical studies conducted thus far have shown mixed results on the protective effect of CHX on the hybrid layer. The study by Araújo et al. [30] investigated whether there was a clinical advantage of the use of $1 \%$ CHX primer over the course of 2 years, while Sartori et al. [31] conducted a 5-year clinical follow up, using a $2 \% \mathrm{CHX}$ primer. Both studies investigated non-carious cervical lesions and neither found a difference in survival in the CHX group as compared to the control group. Non-carious lesions of the $\mathrm{V}$ class are quite specific, there the dentin is hyper-mineralized and structurally different compared to sound or caries-affected dentin, which might have influenced the results. Carrilho et al. [32], on the other hand, found that the use of a CHX primer preserves bond strength in restorations of the I class, after 14 months of intra-oral use. These differences demonstrate the importance of the type of cavity and dentinal substrate in in vivo evaluations, and in general, the importance of standardizing clinical research requirements and methods, and hence making the studies more comparable.

\section{Conclusions}

The CHX-containing adhesive tested in the present study seems to perform better in terms of bond strength preservation and protease inhibition compared to the adhesive system that does not contain CHX. It could be hypothesized that this effect is due to the CHX, but the possibility of the influence of the adhesive system itself must not be completely excluded. Within the limitations of this study, it seems that the use of CHX-blended adhesive could be recommended as a feasible technique in every-day clinical practice, since the inhibitor seems to be efficiently delivered to the dentinal substrate, without prolonging chair time.

\section{Declarations of interest}

None.

\section{Acknowledgments}

The authors wish to thank Mr. Aurelio Valmori for technical assistance. The study was partially supported with MIUR grants (Italy).

\section{References}

[1] D. Pashley, F. Tay, C. Yiu, M. Hashimoto, L. Breschi, R. Carvalho, Collagen degradation by host-derived enzymes during aging, J. Dent. Res. 83 (2004) 216-221.

[2] A. Mazzoni, D.H. Pashley, Y. Nishitani, L. Breschi, F. Mannello, L. Tjäderhane, M. Toledano, E.L. Pashley, F.R. Tay, Reactivation of inactivated endogenous proteolytic activities in phosphoric acid-etched dentine by etch-and-rinse adhesives, Biomaterials 27 (2006) 4470-4476, https://doi.org/10.1016/j.biomaterials.2006. 01.040 .

[3] Y. Nishitani, M. Yoshiyama, B. Wadgaonkar, L. Breschi, F. Mannello, A. Mazzoni, R. Carvalho, L. Tjäderhane, F. Tay, D. Pashley, Activation of gelatinolytic / collagenolytic activity in dentin by self etching adhesives, Eur. J. Oral Sci. 114 (2006) $160-166$.

[4] A. Mazzoni, L. Tjäderhane, V. Checchi, R. Di Lenarda, T. Salo, F.R. Tay, D.H. Pashley, L. Breschi, Role of dentin MMPs in caries progression and bond stability, J. Dent. Res. 94 (2015) 241-251, https://doi.org/10.1177/ 0022034514562833.

[5] A. Mazzoni, F. Mannello, F.R. Tay, G.A.M. Tonti, S. Papa, G. Mazzotti, R. Di Lenarda, D.H. Pashley, L. Breschi, Zymographic analysis and characterization of MMP-2 and -9 forms in human sound dentin, J. Dent. Res. 86 (2007) 436-440, https://doi.org/10.1177/154405910708600509.
[6] A. Mazzoni, V. Papa, F. Nato, M. Carrilho, L. Tjäderhane, A. Ruggeri, P. Gobbi, G. Mazzotti, F.R. Tay, D.H. Pashley, L. Breschi, Immunohistochemical and biochemical assay of MMP-3 in human dentine, J. Dent. 39 (2011) 231-237, https:// doi.org/10.1016/j.jdent.2011.01.001.

[7] J. Santos, M. Carrilho, T. Tervahartiala, T. Sorsa, L. Breschi, A. Mazzoni, D. Pashley, F. Tay, C. Ferraz, L. Tjäderhane, Determination of matrix metalloproteinases in human radicular dentin, J. Endod. 35 (2009) 686-689, https://doi.org/10.1016/j. joen.2009.02.003.

[8] M. Sulkala, T. Tervahartiala, T. Sorsa, M. Larmas, T. Salo, L. Tjäderhane, Matrix metalloproteinase-8 (MMP-8) is the major collagenase in human dentin, Arch. Oral Biol. 52 (2007) 121-127, https://doi.org/10.1016/j.archoralbio.2006.08.009.

[9] M. Sulkala, M. Larmas, T. Sorsa, T. Salo, L. Tjäderhane, The localization of matrix metalloproteinase-20 (MMP-20, enamelysin) in mature human teeth, J. Dent. Res. 81 (2002) 603-607.

[10] H. Palosaari, C. Pennington, M. Larmas, L. Tjäderhane, T. Salo, Expression profile of matrix metalloproteinases (MMPs) and tissue inhibitors of MMPs in mature human odontoblasts and pulp tissue, Eur. J. Oral Sci. 111 (2003) 117-127.

[11] A.R. Hannas, J.C. Pereira, J.M. Granjeiro, L. Tjäderhane, A.R. Hannas, J.C. Pereira, J.M. Granjeiro, L. Tjäderhane, The role of matrix metalloproteinases in the oral environment, Acta Odontol. Scand. 65 (2007) 1-13, https://doi.org/10.1080/ 00016350600963640.

[12] F.D. Nascimento, C.L. Minciotti, S. Geraldeli, M.R. Carrilho, D.H. Pashley, F.R. Tay, H.B. Nader, T. Salo, L. Tjäderhane, I.L.S. Tersariol, Cysteine cathepsins in human carious dentin, J. Dent. Res. 90 (2011) 506-511, https://doi.org/10.1177/ 0022034510391906.

[13] A. Frassetto, L. Breschi, G. Turco, G. Marchesi, R. Di Lenarda, F.R. Tay, D.H. Pashley, M. Cadenaro, Mechanisms of degradation of the hybrid layer in adhesive dentistry and therapeutic agents to improve bond durability - a literature review, Dent. Mater. 32 (2016) e41-e53, https://doi.org/10.1016/j.dental.2015. 11.007.

[14] R. Gendron, D. Grenier, T. Sorsa, D. Mayrand, Inhibition of the activities of matrix metalloproteinases 2, 8, and 9 by chlorhexidine, Clin. Diagn. Lab. Immunol. 6 (1999) 437-439.

[15] P.M.C. Scaffa, C.M.P. Vidal, N. Barros, T.F. Gesteira, A.K. Carmona, L. Breschi, D.H. Pashley, L. Tjaderhane, I.L.S. Tersariol, F.D. Nascimento, M.R. Carrilho, Chlorhexidine inhibits the activity of dental cysteine cathepsins, J. Dent. Res. 91 (2012) 420-425, https://doi.org/10.1177/0022034511435329.

[16] L. Breschi, F. Cammelli, E. Visintini, A. Mazzoni, M. Carrilho, M. Cadenaro, S. Foulger, F.R. Tay, Influence of chlorhexidine concentration on the durability of etch-and-rinse dentin bonds: a 12-month in vitro study, J. Adhes. Dent. 11 (2009) 191-198.

[17] L. Breschi, A. Mazzoni, F. Nato, M. Carrilho, L. Tjäderhane, A. Ruggeri Jr, F.R. Tay, E. De Stefano, Chlorhexidine stabilizes the adhesive interface: a 2 year in vitro study, Dent. Mater. 26 (2010) 1-12, https://doi.org/10.1016/j.dental.2009.11.153. Chlorhexidine.

[18] J. Zhou, J. Tan, L. Chen, D. Li, Y. Tan, The incorporation of chlorhexidine in a twostep self-etching adhesive preserves dentin bond in vitro, J. Dent. 37 (2009) 807-812, https://doi.org/10.1016/j.jdent.2009.06.011.

[19] J. Zhou, J. Tan, X. Yang, C. Cheng, X. Wang, L. Chen, Effect of chlorhexidine application in a self-etching adhesive on the immediate resin-dentin bond strength, $\mathrm{J}$ Adhes. Dent. 12 (2010) 27-31, https://doi.org/10.3290/j.jad.a17543.

[20] J. Zhou, J. Tan, X. Yang, X. Xu, D. Li, L. Chen, MMP-inhibitory effect of chlorhexidine applied in a self-etching adhesive, J. Adhes. Dent. 13 (2011) 111-115, https://doi.org/10.3290/j.jad.a18783.

[21] E.M. da Silva, C.U.F. de Sá Rodrigues, M.P. de Oliveira Matos, T.R. de Carvalho, G.B. dos Santos, C.M. Amaral, Experimental etch-and-rinse adhesive systems containing MMP-inhibitors: physicochemical characterization and resin-dentin bonding stability, J. Dent. 43 (2015) 1491-1497, https://doi.org/10.1016/j.jdent. 2015.10.004.

[22] R. Stanislawczuk, F. Pereira, M.A. Muñoz, I. Luque, P.V. Farago, A. Reis, A.D. Loguercio, Effects of chlorhexidine-containing adhesives on the durability of resin-dentine interfaces, J. Dent. 42 (2014) 39-47, https://doi.org/10.1016/j.jdent. 2013.11.002.

[23] C.K.Y. Yiu, N. Hiraishi, F.R. Tay, M. King, Effect of chlorhexidine incorporation into dental adhesive resin on durability of resin-dentin bond, J. Adhes. Dent. 14 (2012) 355-362, https://doi.org/10.3290/j.jad.a25674.

[24] C. Pomacóndor-Hernández, A. Nogueira, D.G. Antunes, Effect of replacing a component of a self-etch adhesive by chlorhexidine on bonding to dentin, Braz. Dent. J. 24 (2013) 335-339.

[25] C. Sabatini, Effect of a chlorhexidine-containing adhesive on dentin bond strength stability, Oper. Dent. 38 (2013) 609-617, https://doi.org/10.2341/12-239-L.

[26] E.A. Campos, G.M. Correr, D.P. Leonardi, F. Barato-Filho, C.C. Gonzaga, J.C. Zielak, Chlorhexidine diminishes the loss of bond strength over time under simulated pulpal pressure and thermo-mechanical stressing, J. Dent. 37 (2009) 108-114, https://doi.org/10.1016/j.jdent.2008.10.003.

[27] A.D. Loguercio, V. Hass, M.F. Gutierrez, I.V. Luque-Martinez, A. Szezs, R. Stanislawczuk, M.C. Bandeca, A. Reis, Five-year effects of chlorhexidine on the in vitro durability of resin/dentin interfaces, J. Adhes. Dent. 18 (2016) 35-43, https:// doi.org/10.3290/j.jad.a35514.

[28] S. Talungchit, J.L.P. Jessop, D.S. Cobb, F. Qian, S. Geraldeli, D.H. Pashley, S.R. Armstrong, Ethanol-wet bonding and chlorhexidine improve resin-dentin bond durability: quantitative analysis using raman spectroscopy, J. Adhes. Dent. 16 (2014) 441-450, https://doi.org/10.3290/j.jad.a32695.

[29] A.P. Manso, R.H.M. Grande, A.K. Bedran-Russo, A. Reis, A.D. Loguercio, D.H. Pashley, R.M. Carvalho, Can $1 \%$ chlorhexidine diacetate and ethanol stabilize resin-dentin bonds? Dent. Mater. 30 (2014) 735-741, https://doi.org/10.1016/j. 
dental.2014.04.003.

[30] M.S.R.G. Araújo, L.C. Souza, F.M. Apolonio, L.O. Barros, A. Reis, A.D. Loguercio, V.P.A. Saboia, Two-year clinical evaluation of chlorhexidine incorporation in twostep self-etch adhesive, J. Dent. 43 (2015) 140-148, https://doi.org/10.1016/j. jdent.2014.07.010.

[31] N. Sartori, S.C. Stolf, S.B. Silva, G.C. Lopes, M. Carrilho, Influence of chlorhexidine digluconate on the clinical performance of adhesive restorations: a 3-year followup, J. Dent. 41 (2013) 1188-1195, https://doi.org/10.1016/j.jdent.2013.09.004.

[32] M.R.O. Carrilho, S. Geraldeli, F. Tay, M. de Goes, R. Carvalho, L. Tjäderhane, In vivo preservation of hybrid layer by chlorhexidine, J. Dent. Res. 86 (2007) 529-533.

[33] A. Mazzoni, P. Scaffa, M. Carrilho, L. Tjäderhane, R. Di Lenarda, A. Polimeni, A. Tezvergil-Mutluay, F.R. Tay, D.H. Pashley, L. Breschi, Effects of etch-and-rinse and self-etch adhesives on dentin MMP-2 and MMP-9, J. Dent. Res. 92 (2013) 82-86, https://doi.org/10.1177/0022034512467034.

[34] A. Mazzoni, F. Nascimento, M. Carrilho, I. Tersariol, V. Papa, L. Tjaderhane, R. Di Lenarda, F. Tay, D. Pashley, L. Breschi, MMP activity in the hybrid layer detected with in situ zymography, J. Dent. Res. 91 (2012) 467-472, https://doi.org/10. 1177/0022034512439210.

[35] A. Mazzoni, F.M. Apolonio, V.P.A. Saboia, S. Santi, V. Angeloni, V. Checchi, R. Curci, R. Di Lenarda, F.R. Tay, D.H. Pashley, L. Breschi, Carbodiimide inactivation of MMPs and effect on dentin bonding, J. Dent. Res. 93 (2014) 263-268, https://doi.org/10.1177/0022034513516465.
[36] L. Breschi, A. Mazzoni, A. Ruggeri, M. Cadenaro, R. Di Lenarda, E. De Stefano Dorigo, Dental adhesion review: aging and stability of the bonded interface, Dent. Mater. 24 (2008) 90-101, https://doi.org/10.1016/j.dental.2007.02.009.

[37] M. Hashimoto, H. Ohno, H. Sano, M. Kaga, H. Oguchi, In vitro degradation of resindentin bonds analyzed by microtensile bond test, scanning and transmission electron microscopy, Biomaterials 24 (2003) 3795-3803, https://doi.org/10.1016/ S0142-9612(03)00262-X.

[38] M.R. Carrilho, R.M. Carvalho, E.N. Sousa, J. Nicolau, L. Breschi, A. Mazzoni, L. Tjäderhane, F.R. Tay, K. Agee, D.H. Pashley, Substantivity of chlorhexidine to human dentin, Dent. Mater. 26 (2010) 779-785, https://doi.org/10.1016/j.dental. 2010.04.002.

[39] J. Kim, T. Uchiyama, M. Carrilho, K.A. Agee, A. Mazzoni, L. Breschi, R.M. Carvalho, L. Tj??derhane, S. Looney, C. Wimmer, A. Tezvergil-Mutluay, F.R. Tay, D.H. Pashley, Chlorhexidine binding to mineralized versus demineralized dentin powder, Dent. Mater. 26 (2010) 771-778, https://doi.org/10.1016/j.dental.2010. 04.001.

[40] P. Zheng, M. Zaruba, T. Attin, A. Wiegand, Effect of different matrix metalloproteinase inhibitors on microtensile bond strength of an etch-and-rinse and a selfetching adhesive to dentin, Oper. Dent. 40 (2014) 80-86, https://doi.org/10.2341/ 13-162-L.

[41] N. Hiraishi, C.K.Y. Yiu, N.M. King, F.R. Tay, Effect of $2 \%$ chlorhexidine on dentin microtensile bond strengths and nanoleakage of luting cements, J. Dent. 37 (2009) 440-448, https://doi.org/10.1016/j.jdent.2009.02.002. 